\begin{tabular}{c} 
International Journal of Engineering \& Technology, 7 (4) (2018) 2325-2331 \\
International Journal of Engineering \& Technology \\
SPC \\
Website: $\begin{array}{c}\text { www.sciencepubco.com/index.php/IJET } \\
\text { doi: } 10.14419 / \text { ijet.v7it.13619 } \\
\text { Research paper }\end{array}$ \\
\hline
\end{tabular}

\title{
Evaluation of 3d facial paralysis using fuzzy logic
}

\author{
Banita $^{1 *}$, Dr. Poonam Tanwar ${ }^{2}$ \\ ${ }^{1}$ Research Scholar, Lingaya's University, Faridabad \\ ${ }^{2}$ Associate Professor, Manav Rachana University, Faridabad \\ *Corresponding author E-mail: banita861023@yahoo.com
}

\begin{abstract}
Face recognition are of great interest to researchers in terms of Image processing and Computer Graphics. In recent years, various factors become popular which clearly affect the face model. Which are ageing, universal facial expressions, and muscle movement. Similarly in terms of medical terminology the facial paralysis can be peripheral or central depending on the level of motor neuron lesion which can be below the nucleus of the nerve or supra nuclear. The various medical therapy used for facial paralysis are electroaccupunture, electrotherapy, laser acupuncture, manual acupuncture which is a traditional form of acupuncture. Imaging plays a great role in evaluation of degree of paralysis and also for faces recognition. There is a wide research in terms of facial expressions and facial recognition but limited research work is available in facial paralysis. House- Brackmann Grading system is one of the simplest and easiest method to evaluate the degree of facial paralysis. During evaluation common facial expressions are recorded and are further evaluated by considering the focal points of the left or the right side of the face. This paper presents the classification of face recognition and its respective fuzzy rules to remove uncertainty in the result after evaluation of facial paralysis.
\end{abstract}

Keywords: Stages of Face Recognition; 3D Face Recognition; CNN; Evaluation of Facial Paralysis; MAMDANI Model.

\section{Introduction}

Face recognition is a biometric technique used to solve number of unsolved problem in terms of criminal detection or to recognize the face. To identify or verify person identity face recognition is widely used. It can be accessed from video or images of face model.

Face recognition include only three stages:

- Face location detection.

- Feature extraction.

- Facial image classification.

In face location detection facial landmarks have been used to detect the uniqueness of face model. Feature extraction includes preprocessing technique and lastly face image classification detects whether image is in $2 \mathrm{D}$ or in $3 \mathrm{D}$ imaging.

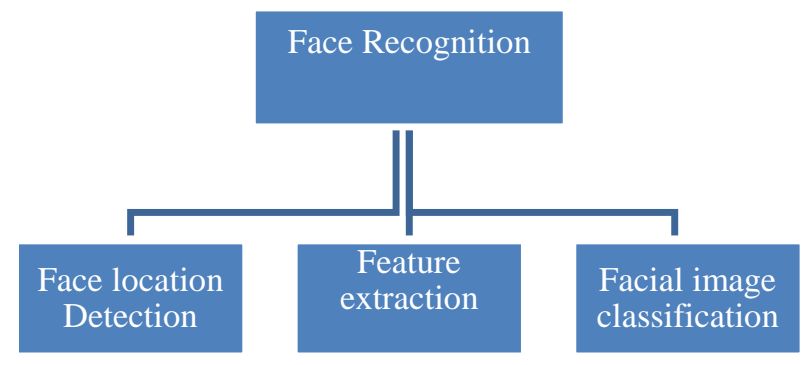

Fig. 1: Stages of Face Recognition.

\section{3-Dimensional face recognition}

It is strictly deals with the shape (Dimensions) of a face model. One of the most common advantage of using 3D face recognition it that during analysis there is no effect on the outcomes with the variation in lightning or pose.3D technique in face recognition includes various data points (Fiducial landmarks) on face image to get more precise results. While combining various techniques in 3D face recognition, 3D is independent to facial expression like smiling.

Face recognition is quite useful in detecting crime. Here image of face can be captured at real time or at rest without touching a person which help for recognizing a person. It is similar to biometric process but3D imaging deals with more accuracy as compared with biometric process. Like biometric identification Face recognition is widely used and such applications are used in mobile phones as well to make more precise results.

As P. Jonathon Phillips et.al. in 2006 clearly define the Face Recognition Grad Challenge (FRGC)on the basis of identification and verification that for identification process only single image of face is required to identify a person, whereas for verification process in face recognition two or more face images are required in dataset. It is further concluded that FRGC in 3 Dimensional imaging identification and verification depends on range and the texture Channel. The distance from where an image has been captured and the texture of image detects the identification of a person.

P. Jonathon Phillips et.al. Further introduced three possible goals:

- High resolution still imagery.

- Multiple still images.

- 3D facial imagery. 
3D face recognition algorithm used to detect face model from the 3D images which has been saved in the database. It further improves the performance and accuracy in terms of result when compared with 2 Dimensional imaging. In 2D imaging results may vary as it depends on the lightning and pose variation. Moreover $2 \mathrm{D}$ face recognition includes $2 \mathrm{D}$ face images whereas $3 \mathrm{D}$ imaging includes 3D face model images for analysing the different perspectives in terms of face recognition.

For validation and motivation of research in better face recognition under illumination variation in terms of computation and recognition rate Principal Component Analysis has been performed to get relevant results.

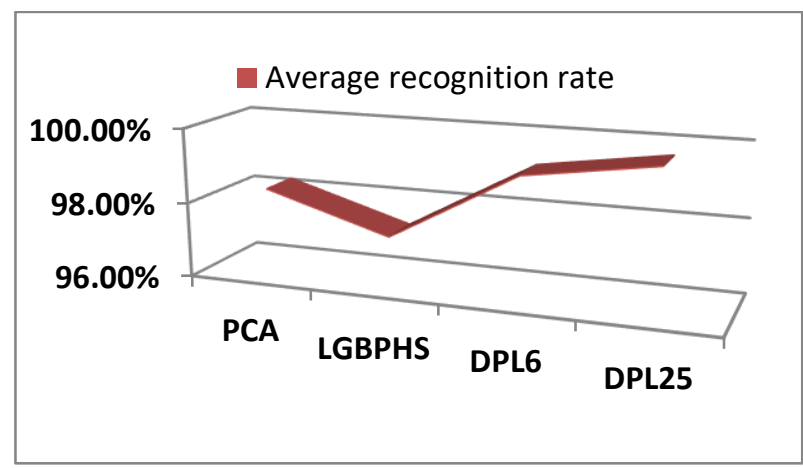

Fig. 2: Average Recognition Rate.

PCA is the well-known popular and successful holistic method [1]. Local Gabor Binary pattern Histogram Sequence (LGBPHS) is a non-statistical based face recognition approach [1].During implementation DPL6 and DPL25 dual stage algorithm has been used which depend on the number of images supplied [1]. Fig.2 clearly determines the average recognition rate in terms of PCA LGBPHS and also dual stage algorithm DPL6 and DPL25. DPL gives better recognition rate than PCA and LGBPHS [1].

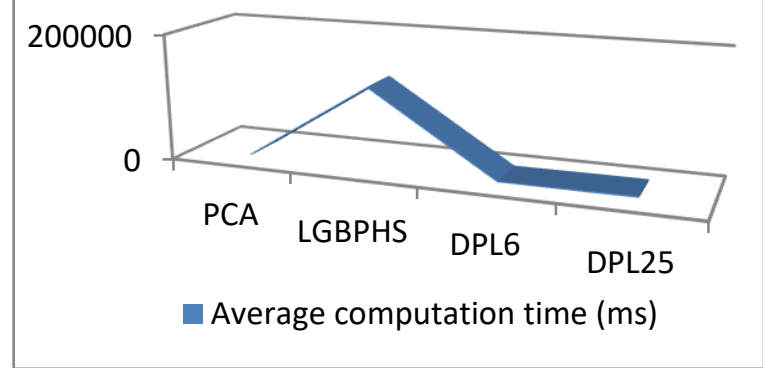

Fig. 3: Average Computation Time (MS).
Here the average computation time per image has been reduced while comparing it with recognition rate [1].Fig.3 clearly defines the average computation time in ms w.r.t. PCA, LGBPHS, DPL6 and DPL25.

\section{Convolution neural networks}

Convolution Neural Networks (CNN) includes a multilayer perception which is designed for minimum pre-processing time. CNN is also known as Shift variant. It includes number of hidden layers along with input and output layer.

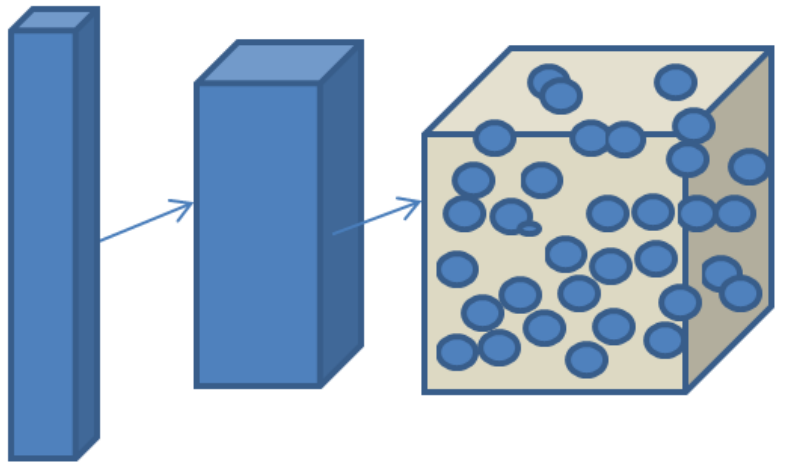

Fig. 4: CNN Layers in 3 Dimensions.

CNN basically includes three categories:

- Pooling.

- Fully Connected.

- Weights.

Pooling can be local or global pooling. It connects the output layer of one neuron to a single neuron in its next layer. Fully connected includes a zigzag architecture because all the neurons are connected to all the neurons present in all the layers. Weights can be shared in convolution layer as neurons are partially or completely connected with each other. Hence best category i terms of time constraints is fully connected CNN because in fully connected $\mathrm{CNN}$ number of processing has been done parallel. In terms of face recognition $\mathrm{CNN}$ is widely used but it again depends on the number of images in database.

Eigen Face is the principal component of initial stage of face recognition. Eigen face is defined as the method to split face image in to number of small set in database. Face recognition depends upon the clarity of face image. For this high resolution digital cameras have been used to recognize face. 


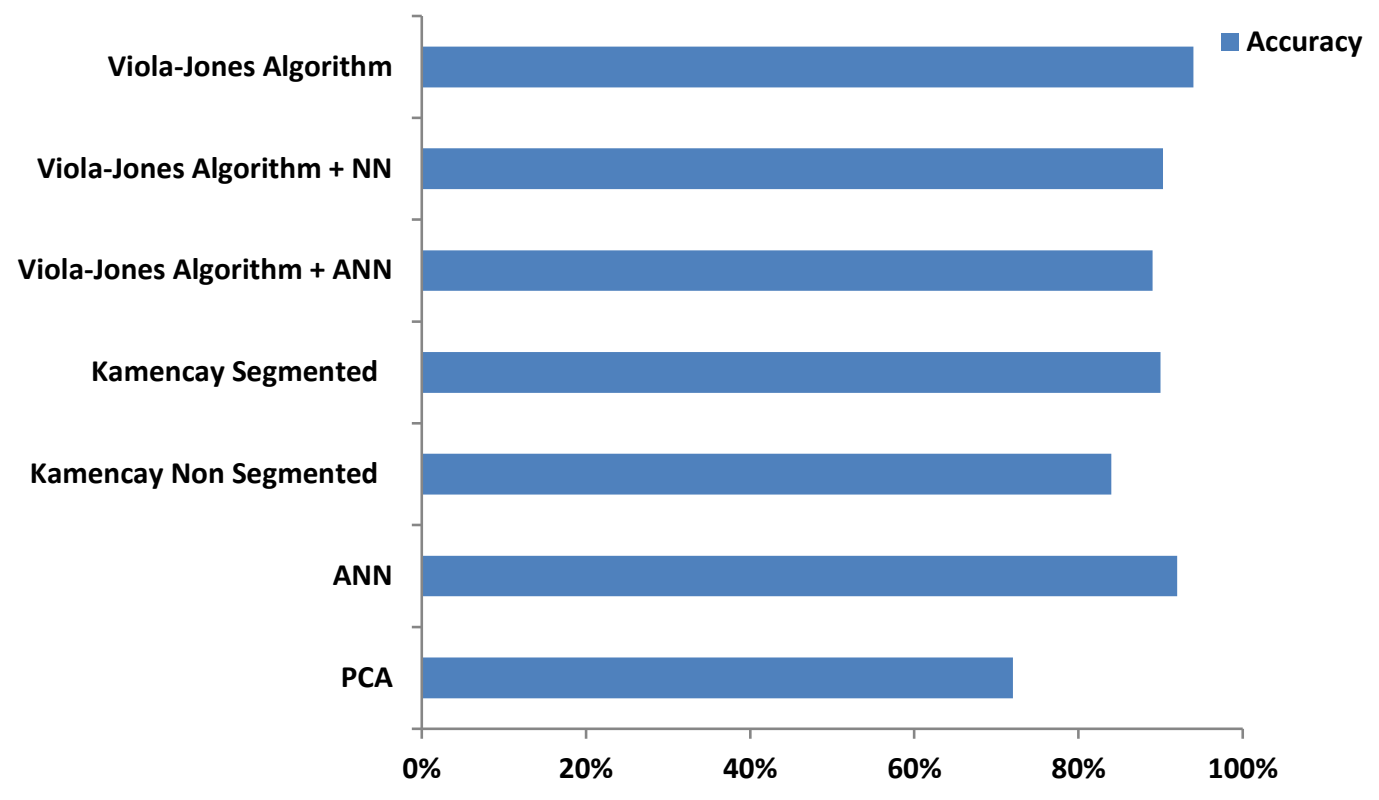

Fig. 5: Face Recognition Techniques and Its Accuracy.

In total five techniques has been compared in terms of accuracy. It has been clearly observed that Viola-Jones Algorithm along with PCA and ANN shows better accuracy in face recognition [6]. Fig.5 represents the accuracy rate in terms of face recognition, hence to identify a person in an image with other techniques Viola-Jones algorithm along with PCA and ANN has been used.

\section{Evaluation of facial paralysis}

Table 1: Classification of Grading by House-Brackmann Grading System (HBGS)

\begin{tabular}{|c|c|c|}
\hline $\begin{array}{l}\text { Grad } \\
\mathrm{e}\end{array}$ & Description & Characteristics \\
\hline I & Normal & Normal Facial function \\
\hline II & $\begin{array}{l}\text { Mild Dysfunc- } \\
\text { tion }\end{array}$ & $\begin{array}{l}\text { Slight synkinesis, weakness, slight asym- } \\
\text { metry }\end{array}$ \\
\hline III & $\begin{array}{l}\text { Moderate } \\
\text { Dysfunction } \\
\text { Moderately }\end{array}$ & Slightly weak with maximum effort \\
\hline IV & $\begin{array}{l}\text { Serve Dys- } \\
\text { functioning }\end{array}$ & Disfiguring Asymmetry \\
\hline V & $\begin{array}{l}\text { Severe Dys- } \\
\text { functioning }\end{array}$ & Asymmetry at Rest \\
\hline VI & $\begin{array}{l}\text { Total Paraly- } \\
\text { sis }\end{array}$ & Nonfunctioning of muscles \\
\hline
\end{tabular}

One of the best approaches to evaluate the level of facial paralysis is House-Brackmann Grading system; it clearly detects the level of facial paralysis with the help of six Universal expressions. HBGS include the grading levels; lower the grading level means the region is less affected. Patients has to performs a series of facial expressions which will further evaluate a grade of Paralysis ranging from Grade I to Grade VI in HBGS. Grade -I represent the normal functioning of face during facial expressions. Grade IIGrade V represent the facial paralysis can be recovered or cannot be recovered. Grade-VI represents total facial paralysis which cannot be recovered.

In case of Bell's palsy face has been split into two halves for comparison between both sides of face model. In case of facial palsy face model has been divided into four quadrants to evaluate the branch of facial nerve i.e. VIIth Cranial nerve. For the evaluation purpose in 3D fuzzy logic plays a great role to achieve high accuracy when compared with $2 \mathrm{D}$ imaging. Questionnaire has been used to evaluate and to judge whether patient need further medical surgery or not.

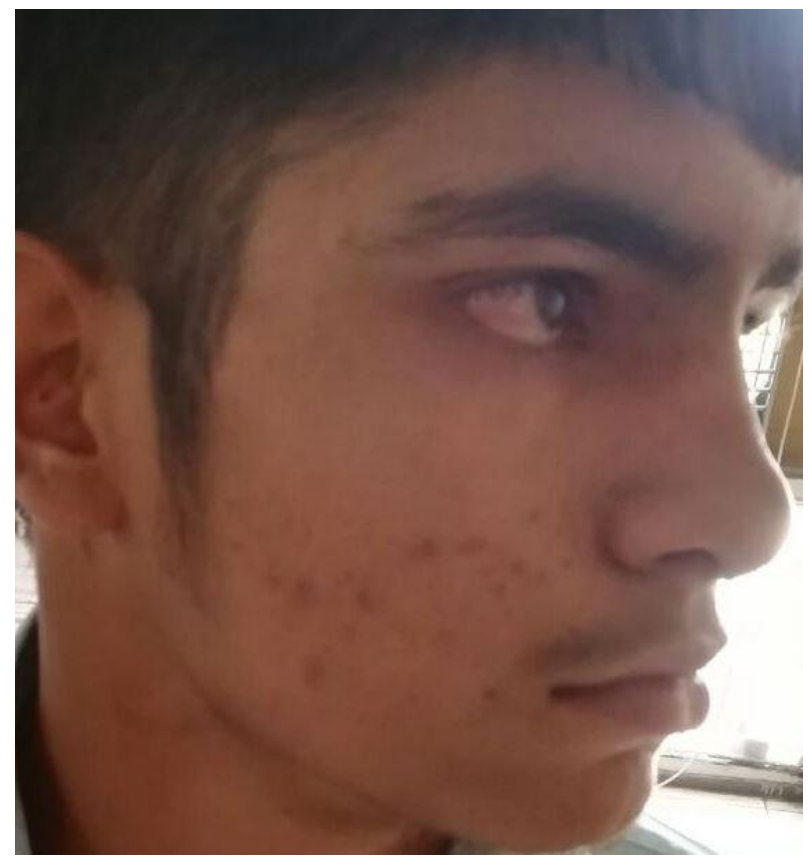

Fig. 6: Right Side of Face Model.

In terms of 3D imaging for analysis of facial paralysis large data has been evaluated within an appropriate time. As 3D imaging only deals with the depth of the image hence for evaluating the level of severity for facial paralysis two images has been captured for each patient for ear-to-ear coverage of face model. Fig. 6 clearly represents the right side of face to analyze the Grade of Facial paralysis as per HBGS grading system. Here image has been captured during facial expression but due to affected side of face model no change has been notified during facial expression i.e. closing of eyes.

To make result more accurate fuzzy set of rules has been used in the uncertainty result where it results as may or may not be cured. In this category result has been found at high risk for which to remove this uncertainty fuzzy set of rules has been applied.

Depending on the distance using Euclidian distance evaluation in 3D the difference as outcome has been categorized into 3 sections;

- Facial Paralysis patient can be cured.

- Facial paralysis cannot be cured.

- Facial paralysis patient may or may not be cured. 
Out of above three categories the outcome where "Facial paralysis patient may or may not be cured" deals with uncertainty in result. Hence to overcome such uncertainty fuzzy logic has been used for further evaluation.

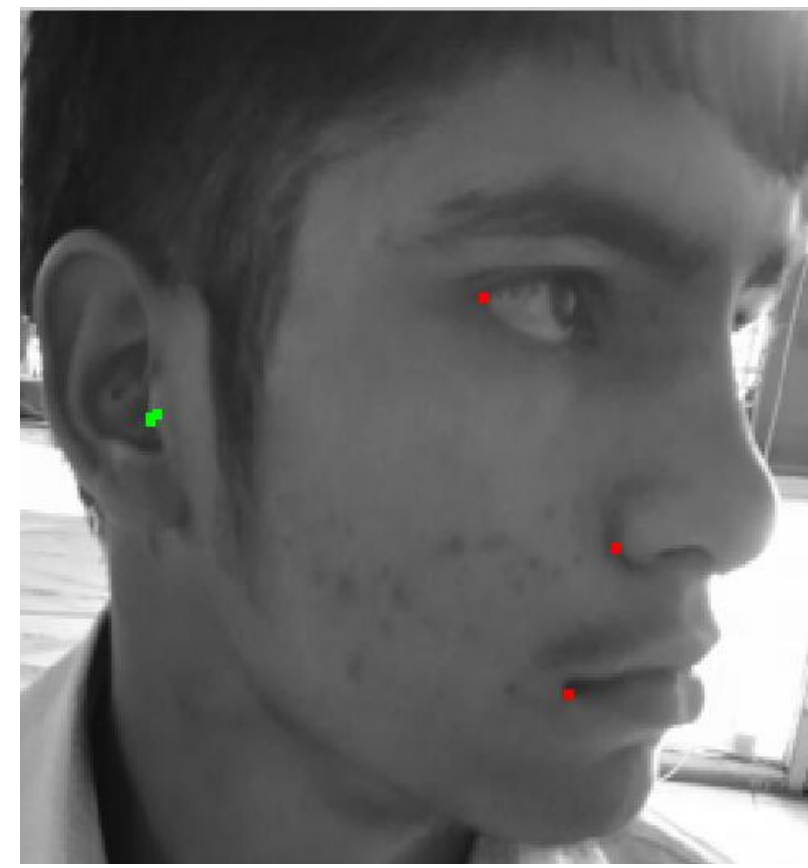

Fig. 7: After Implementation of Right Side of Face Model.

After image storage MATLAB has been used to detect the distance of facial regions with the help of Fiducial landmarks. To make the evaluation more accurate and precise Fig.7 consists of two different colors has been used in facial landmarks. Two main points' i.e. Key points and its respective reference points has been taken for evaluating the degree of facial paralysis. Change in facial dimensions results in facial asymmetry which is not constant in all cases. Traditionally Medical image technology researchers have been using Two-Dimensional (2D) medical imaging and then reconstructing them into Three-Dimensional (3D) for analyzing the image for better result quality.

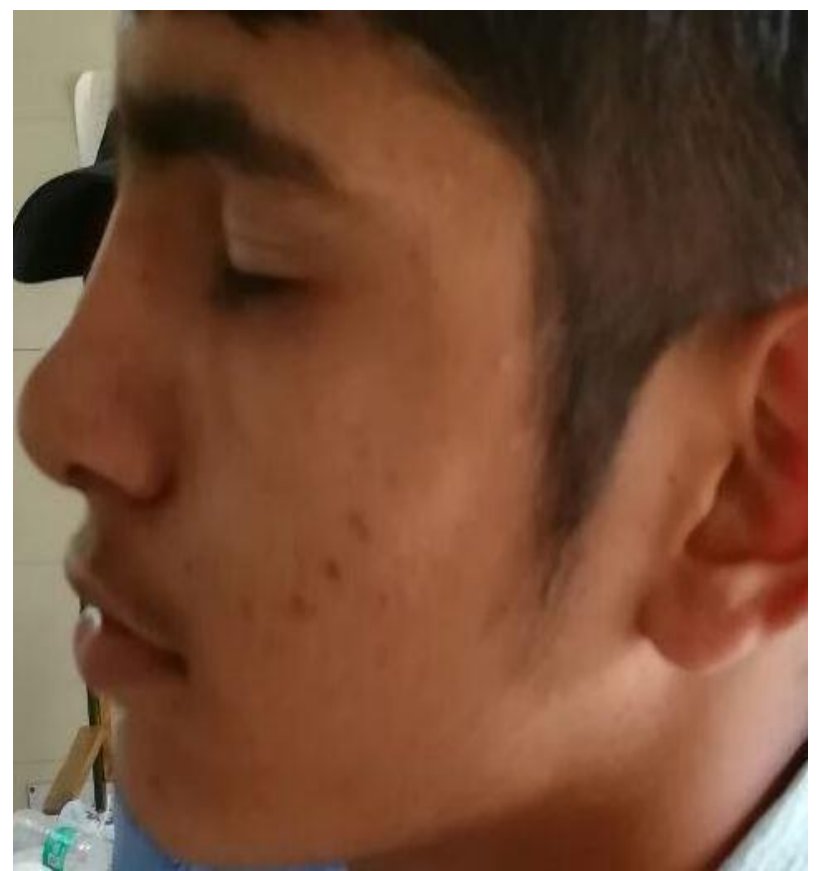

Fig. 8: Left Side of Face Model.

Now for same patient left side of face image has been captured such that to detect the variation in the distance of facial regions. Fig. 8 clearly represents the left side of face to analyze the Grade of Facial paralysis as per HBGS grading system. Here image has been captured during facial expression but due to healthy side of face model change has been notified during facial expression i.e. closing of eyes.

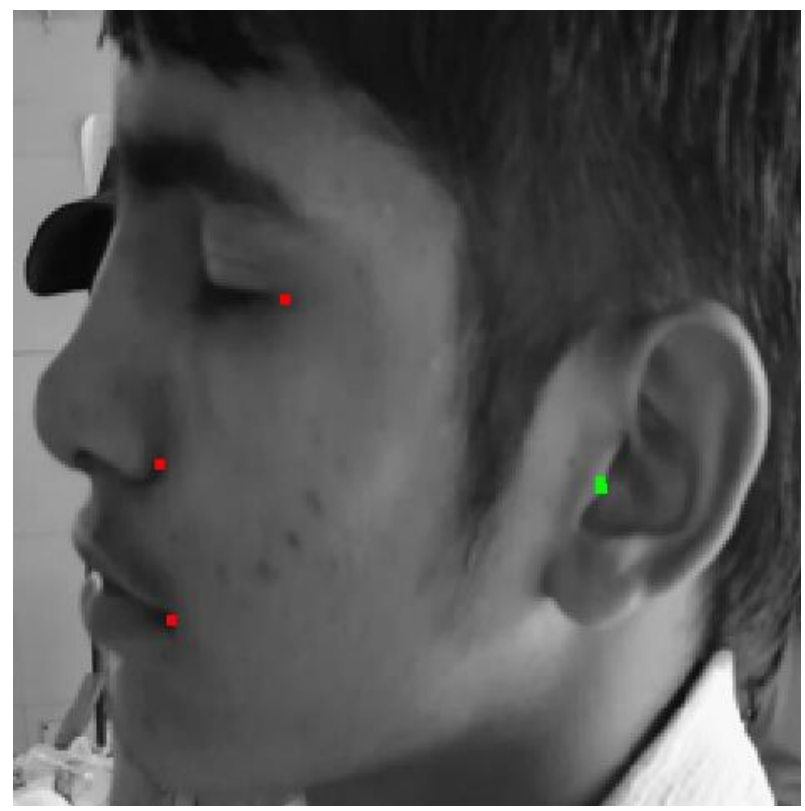

Fig. 9: After Implementation of Left Side of Face Model.

After image storage MATLAB has been used to detect the distance of facial regions with the help of Fiducial landmarks. To make the evaluation more accurate and precise Fig.9 represents two different colors which has been used in facial landmarks. Two main points' i.e. Key points and its respective reference points has been taken for evaluating the degree of facial paralysis

Euclidian distance in terms of $2 \mathrm{D}$ and in $3 \mathrm{D}$ includes face model and distance evaluation using $\mathrm{X}-\mathrm{Y}$ and $\mathrm{X}-\mathrm{Y}-\mathrm{Z}$ axis respectively. During evaluation classifier has been used for further outcomes at great accuracy. Proposed method worked on 3D imaging to evaluate the severity of facial paralysis. It has been notified that if difference in distance is $50-80 \mathrm{~mm}$ using Euclidian distance evaluation then uncertainty has been detected which reduces the accuracy. But to improve the accuracy of proposed method fuzzy logic has been implemented.

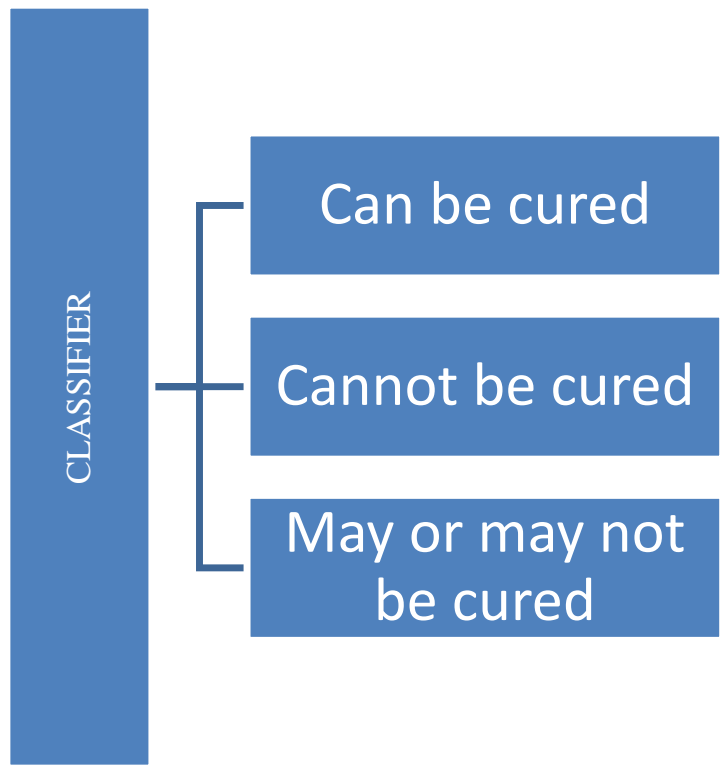

Fig. 10: Classifier for Evaluation of Facial Paralysis.

Classifier has been used during evaluation of facial paralysis to categories the certainty and uncertainty in result in terms of $2 \mathrm{D}$ as well as for 3D imaging.Fig.10 represent the 3 categories of classi- 
fier based on appropriate range in Euclidian distance. If the variation in distance after comparison of left and right side of face model has been less than $50 \%$ then there must be chances to be cured, if difference in facial landmarks along left and right side of face has been more than $80 \%$ than patient cannot be able to cure from facial paralysis i.e. there must be some variation on face dimensions after treatment as well.

But for the category "May or may not be cured" one different technique has been implemented to remove the uncertainty in terms of evaluation of facial paralysis.

If the difference is $0-50 \mathrm{~mm}$ then patient can be cured, if difference is $80-100 \mathrm{~mm}$ then the patient cannot be cured completely but somehow recovery has been notified. Whereas between $50-80 \mathrm{~mm}$ difference in distance leads to the result as "Patient may or may not be cured". It clearly defines the risk factor after evaluation of distance in Euclidian distance evaluation in 3D.

The colored portion is the value at which facial paralysis evaluation results in risk factor. That shaded portion includes further evaluation using fuzzy set of rules to minimize the risk factor and to get more precise result. Questionnaire has been further investigated to follow-up the progress rate of facial paralysis patients. Additional in same in terms of medical science Electromyography (EMG) has been used to evaluate the severity of facial paralysis but not the recovery rate of facial paralysis patient. Whereas in EMG current has been passed on the facial regions and its respective action which has been performed to detect whether signal have been passed upto the mark or not has been recorded with the help of electrode.

All the waveforms has been recorded in terms of medical science for just to detect whether there is some weakness on facial nerve or not. But it will not detect whether patient can be cured or cannot be cured after prescription. To overcome from such diagnosis of facial paralyzed patient fuzzy logic MAMDANI Model has been used to analyze the chances of patient to be cured in outcome.

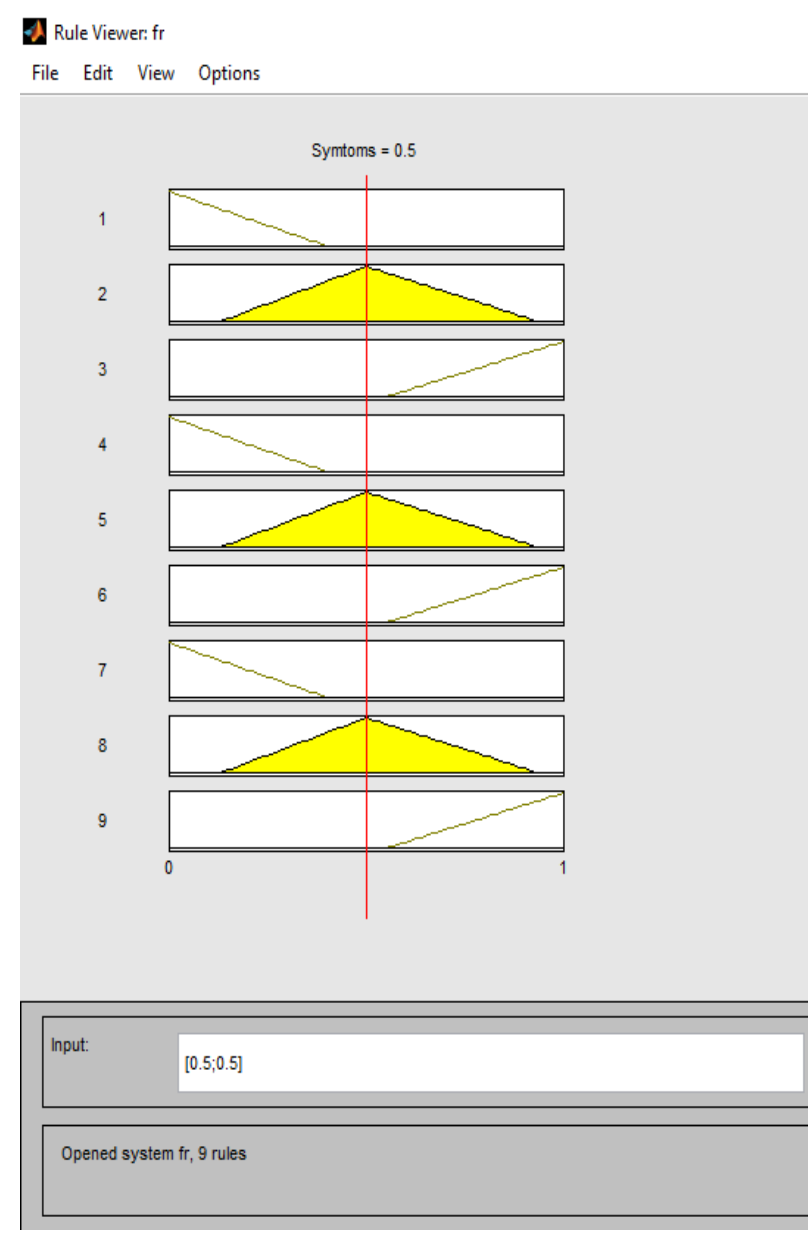

Fig. 11: Fuzzy Rules for Facial Paralysis.
In MAMDANI Model total set of fuzzy rules has been implemented for more accurate result which clearly detects the chances to get recover from facial paralysis.

The proposed model is based on the distance evaluation and the questionnaire which has been evaluated at PGIMS Rohtak after taking the consent of facial paralysis patients.

Total of 47 patients examined under questionnaire and in total of 82 patients evaluated using distance evaluation in 3D imaging.

MAMDANI Model is the first system used to control the uncertainty in any perspective during evaluation. It results in 2 aspects whether value is 0 or 1 . Fig. 11 indicates that when there is no progress rate found then it results in 0 values and if some progress rate has been noted then model results to value1. For facial paralysis patient after questionnaire, progress rate has been followed in different aspects as per the level of facial paralysis.

MAMDANI Fuzzy inference model help to Analyse the evaluation more precisely and quickly. With the help of fuzzy set of rules in different conditions it results in more accurate value.

MAMDANI Model includes 4 basic steps:

a) Fuzzification: In includes the dataset and rules information for input variables.

b) Rule Evaluation: All the fuzzy rules which has been applied during analysis must be stored and evaluated in this category of MAMDANI Model.

c) Aggregation: This category of MAMDANI Model includes the condition implemented in fuzzy set of rules.

d) Defuzzification: Defuzzification is the final step to evaluate all the fuzzy rules. Defuzzification is also defines as the aggregate output fuzzy sets in a single number.

Table 2: Follow-Up for Analyzing Facial Paralysis

\begin{tabular}{llll}
\hline Current state & After 7 days & After 30 days & Progress Rate \\
\hline L & L & L & 0 \\
L & M & M & 0 \\
L & H & M & 1 \\
L & H & H & 1 \\
L & M & H & 1 \\
\hline
\end{tabular}

Here $\mathrm{L}, \mathrm{M}$ and $\mathrm{H}$ is represented as below:

$\mathrm{L}=$ Low progress

$\mathrm{M}=$ Moderate progress

$\mathrm{H}=$ High progress rate

These 3 perspectives is basically the comparison of distance evaluation in MATLAB and the progress rate to reach high accuracy. The proposed method clearly detects the exact progress rate of facial paralysis. One of the major advantage of proposed method is there is no use of facial paralysis for evaluating the level of facial paralysis and the exact progress rate which also help in detection of recovery rate of facial paralysis patient.

As the progress rate has been divided into 3 sections hence in total of 9 set of fuzzy rules has been developed from a single questionnaire. Same has been implemented on all the patients after their consent for evaluation at PGIMS Rohtak. 


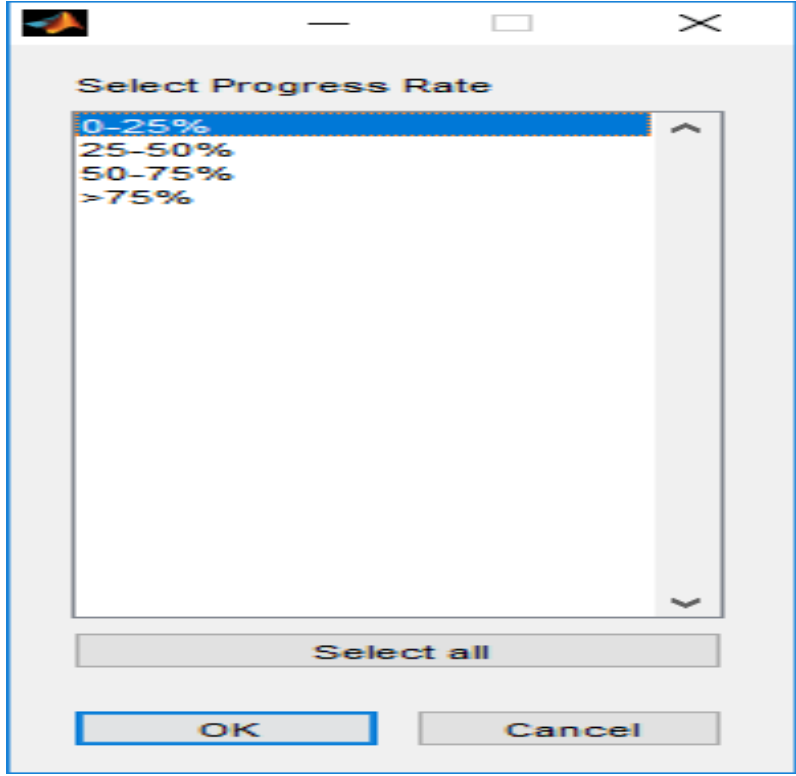

Fig. 12: Progress Rate in 3D.
Progress rate is directly proportional to the recovery of patient from facial paralysis. If the progress rate is low then there will be fewer chances for recovery. Fig. 12 indicates that progress rate is divided into 3 categories i.e. $0-25 \%, 25-50 \%, 50-75 \%$ and greater than $75 \%$.

In case of facial paralysis, medical science is lagging in such field to analyze the recovery rate of paralysis. Progress rate has been evaluated manually as well as physically to select the progress rate. Out of 3 different range of progress depending upon the progress rate proposed method results in the Chances to get cured from facial paralysis in terms of percentage. Progress rate has been used only when there is uncertainty in the result of evaluating facial paralysis. Uncertainty represents that "may or may not be cured", in this section questionnaire has been used manually to follow-up the patient after 7 days or 30 days.

Table 3: Comparison between Various Techniques Used for Face Recognition and Their Outcomes

\begin{tabular}{|c|c|c|c|}
\hline S. No. & Work & Methodology Used & Outcomes \\
\hline 1 & Issam Dagher et.al. Vol.7, Issue:1, 2014 [2] & - $\quad \mathrm{K}$-means clustering algorithm. & $\begin{array}{l}\text { Using SIFT most representative } \\
\text { images is presented in terms of face } \\
\text { recognition. }\end{array}$ \\
\hline 3 & Avenir K.Troitsky, Vol.11 No.6,2016.[4] & $\begin{array}{l}\text { - "Weight" to eye and Bridge to nose } \\
\text { point. }\end{array}$ & $\begin{array}{l}\text { Computation time is decreased by } \\
\text { assigning some "weight" value for } \\
\text { each position. } \\
\text { High probability of face detection. }\end{array}$ \\
\hline 4 & Byoung Chul Ko, January 2018. [5] & $\begin{array}{l}\text { - } \quad \text { Facial action coding system. } \\
\text { - } \quad \text { Facial action unit. }\end{array}$ & $\begin{array}{l}\text { It can be used as a dictionary to new } \\
\text { researchers in FER. }\end{array}$ \\
\hline 5 & $\begin{array}{l}\text { Narayan T. Deshpande, Vol.10,No.5, } \\
\text { 2017.[6] }\end{array}$ & $\begin{array}{l}\text { - Voila-Jones algorithm. } \\
\text { - } \quad \text { Haar- features in Viola-Jones. } \\
\text { - } \text { Face Tagging. }\end{array}$ & $\begin{array}{l}\text { Better accuracy in face recognition } \\
\text { with the proposed method. }\end{array}$ \\
\hline 6 & $\begin{array}{l}\text { Manjunatha Hiremath, Vol.12,No.1, January } \\
2018 \text { [7] }\end{array}$ & $\begin{array}{l}\text { - } \quad \text { PCA, LDA. } \\
\text { - } \quad \text { Elastic bunch graph matching. }\end{array}$ & $\begin{array}{l}\text { Overview of different face recogni- } \\
\text { tion approaches. }\end{array}$ \\
\hline 7 & Xiangyi Kong,et.al., 2018.[8] & $\begin{array}{l}\text { - } \quad \text { Convolution neural networks. } \\
\text { - } \quad \text { Kachine learning. } \\
\text { K-N ear Neighbors. }\end{array}$ & $\begin{array}{l}\text { Artificial Intelligence can automati- } \\
\text { cally detect acromegaly with high } \\
\text { sensitivity and specificity at early } \\
\text { detection. }\end{array}$ \\
\hline 8 & Hwai-Jung Hsu,et.al. [9] & $\begin{array}{l}\text { - True positive rate. } \\
\text { - } \quad \text { False positive rate. } \\
\text { Haar face recognition. }\end{array}$ & $\begin{array}{l}\text { Face recognition technologies are } \\
\text { able to perform adequately on } \\
\text { drones. }\end{array}$ \\
\hline 9 & Raunak Dave,et.al., March 2018.[10] & $\begin{array}{l}\text { - Local region principal component } \\
\text { analysis (LRPCA). } \\
\text { - SVM , Cohort Linear Discriminant } \\
\text { analysis (Cohort LDA) }\end{array}$ & It's the survey of face recognition. \\
\hline 10 & Sudha Narang, Vol.8, No.2, Frb.2018.[11] & $\begin{array}{l}\text { - } \quad \text { Viola-Jones Algorithm. } \\
\text { - } \quad \text { Fisher face } \\
\text { - } \quad \text { Local Binary histogram algorithm. }\end{array}$ & $\begin{array}{l}\text { Tradeoff exists between correct } \\
\text { recognition rate and threshold value. }\end{array}$ \\
\hline 11 & STM Siregar , et.al., 2018.[12] & $\begin{array}{ll}\text { - } & \text { PCA } \\
\text { - } & \text { Face recognition eigen face method. }\end{array}$ & $\begin{array}{l}\text { Pre-processing phase must be done } \\
\text { to get similar image source. }\end{array}$ \\
\hline 12 & Claire L.Witham, July 2017,Elsevier.[13] & $\begin{array}{l}\text { - Local binary pattern } \\
\text { Local discriminant classification algo- } \\
\text { rithm }\end{array}$ & $\begin{array}{l}\text { Possibility to identify individual } \\
\text { macaques by their faces in videos. }\end{array}$ \\
\hline 13 & Suparna Biswas, et.al., 2017.[14] & $\begin{array}{l}\text { - Contourlet transformation } \\
\text { - } \quad \text { Curvelet transformation. }\end{array}$ & $\begin{array}{l}\text { Experimental analysis demonstrate } \\
\text { good performance of proposed meth- } \\
\text { od on data set ORL, JAFFE and } \\
\text { FERET which are available publical- } \\
\text { ly. }\end{array}$ \\
\hline 14 & $\begin{array}{l}\text { Pratibha Sukhija, ett.al., (CMS2016),Science } \\
\text { direct.[15] }\end{array}$ & $\begin{array}{ll}- & \mathrm{PCA} \\
- & \mathrm{LDA}\end{array}$ & $\begin{array}{l}\text { Face recognition rate is better in } \\
\text { proposed model. }\end{array}$ \\
\hline
\end{tabular}




\section{Conclusion}

In this paper, Scale Invariant Feature Transform gave the best recognition result [2]. 3D imaging has been used to upgrade the accuracy because 2D based analysis has difficulty handling large variation in pose and subtle facial behavior [5]. As far as the accuracy in different techniques of face recognition is concern the performance and accuracy of Viola-Jones algorithm, Artificial Neural Network and PCA together is better as compared with other face recognition techniques. Various types of challenges in face recognition can be solved using different algorithm and datasets of face expressions and face detection. Commonly used PCA technique for face recognition but when compared to LDA, LDA consumes less time as compared to PCA. Face recognition has been used in attendance system to make results more precise and accurate. Using face recognition no duplicity has been observed which further help to reduce the error up to its maximum extent. 2D face recognition requires facial expressions whereas in 3D face recognition facial expression is not mandatory. One can evaluate the identity of person without using its respective facial expression in 3D. Lightning variation and pose variation whereas in 3D imaging outcomes may vary with respect to lightning variation and pose variation.Pose variation and lightning has no effect on the outcomes of 3D imaging. Hence 3D imaging has been used for evaluation of facial paralysis. To remove uncertainty up to great extent fuzzy logic sets has been used for minimizing the risk factor. MAMDANI model has been used on risk factor. Euclidian distance in terms of 3D has been used.

Questionnaire has been used for follow-up of the facial paralysis patient. Total of 47 patients examined under questionnaire and in total of 82 patients evaluated using distance evaluation in 3D imaging at different Civil Hospital in Haryana. The proposed approach overcome the problem of light and pose variation and also summarize the data within a standard size of image. Method has been implemented in terms of 3D analysis to detect the chances of patient to be cured.

It has been further concluded that there is no such use of face recognition as the image has been captured using digital camera along both side of face for distance evaluation. It has been further concluded that after implementation fuzzy logic and MAMDANI Model plays a great role to remove the uncertainty in result of evaluation of facial paralysis in terms of 3D imaging which has been a great challenge in 2D imaging. Further it has been concluded that with the help of medical science and 2D analysis an implementation in 3D plays a great role to make the result more accurate. $99.7 \%$ of accuracy has been achieved in 3D for evaluating the level of severity and the chances to recover from facial paralysis which is greater than in 2D.Clear result in 2D is $40 \%$ i.e patient can be cured or patient cannot be cured whereas $60 \%$ result in 2D has been notified as uncertainty in result i.e. "patient can be or cannot be cured". To remove such uncertainty MAMDANI Model has been used in 3D and ear to ear coverage of face has been captured with the help of digital camera for evaluation of facial paralysis.

\section{Acknowledgment}

This work was supported by Pathology and Neurology Department of PFIMS Rohtak, INDIA since 2016.

\section{References}

[1] Hyunjong Cho et.al. "An efficient hybrid face recognition algorithm using PCA and GABOR wavelets", International Journal of advanced Robotics systems, 2014. https://doi.org/10.5772/58473.

[2] Issam Dagher et.al. "Face recognition using the most representative SIFT Images", International Journal of Signal processing, image processing and pattern recognition, Vol.7, Issue: 1, 2014.
[3] Aruni RoyChowdhury "One to many face recognition with Bilinear CNNs",IEEE Conference on application of computer Vision, Published in March 2016.

[4] Avenir K.Troitsky, "Two-level multiple face detection algorithm based on Local feature Search and structure recognition methods", International journal of applied engineering research, Vol.11 No.6, 2016.

[5] Byoung Chul Ko, "A brief review of facial emotion recognition based on visual information" Sensors", January 2018. https://doi.org/10.3390/s18020401.

[6] Narayan T. Deshpande "Face detection and recognition using Viola-Jones algorithm and fusion of PCA and ANN", Advances in Computational sciences and technology", Vol.10, No.5, 2017.

[7] Manjunatha Hiremath, "Current trends on face recognition methods in face biometrics" International journal of computer engineering and applications, Vol.12, No.1 January 2018.

[8] Xiangyi Kong,et.al., "Automatic detection of Acromegaly from facial photographs using machine learning methods" "EBioMedicine" 2018. https://doi.org/10.1016/j.ebiom.2017.12.015.

[9] Hwai-Jung Hsu,et.al . "Face recognition on Drones: Issues and limitations",Institute of Information Science,Academic Sinica.

[10] Raunak Dave,et.al. "Face recognition techniques: A survey", March 2018.

[11] Sudha Narang, "Comparison of face recognition algorithms using Opencv for attendance system" International journal of scientific and research publication, Vol.8, No.2, Feb.2018.

[12] STM Siregar, et.al "Human face recognition using Eigen face in cloud computing environment", 10th International conference $\mathrm{Nu}$ merical analysis in engineering, 2018.

[13] Claire L.Witham, "Automated face recognition of rhesus macaques" Journal of neuroscience method, July 2017, Elsevier.

[14] Suparna Biswas, et.al. "An efficient face recognition method using contourlet and curvelet transform", Journal of king saud UniversityComputer and information science, 2017.

[15] Pratibha Sukhija, et.al. "Face recognition system using genetic algorithm", International conference on computational modelling and security (CMS2016), Science direct. https://doi.org/10.1016/j.procs.2016.05.183. 